\title{
CELL PROTEINS BIND TO A 67 NUCLEOTIDE SEQUENCE WITHIN THE 3' NONCODING REGION (NCR) OF SIMIAN HEMORRHAGIC FEVER VIRUS (SHFV) NEGATIVE-STRAND RNA
}

\author{
You-Kyung Hwang and Margo A. Brinton \\ Department of Biology \\ Georgia State University \\ Atlanta, Georgia 30303
}

\section{ABSTRACT}

The 3'NCR of the SHFV negative-strand RNA [SHFV 3'(-)NCR RNA] is thought to be the initiation site of full-length and possibly also subgenomic positive-strand RNA and so is likely to contain cis-acting signals for viral RNA replication. Cellular and viral proteins may specifically interact with this region to form replication complexes. When in vitro transcribed SHFV 3'(-)NCR RNA was used as a probe in gel mobility shift assays, two RNA-protein complexes were detected with MA104 S100 cytoplasmic extracts. The specificity of these RNA-protein interactions was demonstrated by competition gel mobility shift assays. Four MA104 proteins $(103,86,55$, and $36 \mathrm{kDa})$ were detected by UV-induced cross-linking assays and three proteins $(103,55$, and $36 \mathrm{kDa})$ were detected by northwestern blotting assays. The binding sites for these proteins were mapped to the region between nucleotides 117 to 184 on the SHFV 3'(-)NCR RNA. Four cellular proteins with identical molecular masses to those of the proteins that bind to the SHFV 3'(-)NCR RNA were detected by the 3'(-) NCR of another arterivirus, LDV-C, suggesting that divergent arteriviruses utilize the same set of conserved cell protein domains.

\section{INTRODUCTION}

Simian hemorrhagic fever virus (SHFV) causes a fetal hemorrhagic disease in macaque monkeys. SHFV-infected macaques develop early fever, mild facial edema, anorexia, dehydration, proteinuria, cyanosis, skin petechia, bloody diarrhea, nose bleeds, 
hemorrhages in the skin, and occasional bleeding in the orbits of the eyes. Death occurs within 1 to 2 weeks after SHFV infection (London, 1977). In contrast, SHFV causes a persistent infection but no disease in chimpanzees, African green monkeys, and baboons (Gravell et al., 1980; London, 1977). These species are thought to be natural hosts for SHFV.

SHFV is a positive-strand RNA virus and a member of the family Arteriviridae. Although the organization and replication strategy of the arterivirus genome are similar to those of coronaviruses, arteriviruses differ from coronaviruses in their virion morphology and genome size (Plagemann, 1996). The genome of SHFV is approximately 14.5 kilobases in length (Sagripanti, 1984; Brinton et al., unpublished data; Smith, 1997) and contains a type I cap at the 5' end (Sagripanti et al., 1986) and a poly (A) tract at the 3' end (Sagripanti, 1984). The SHFV genome encodes a large, nonstructural polyprotein ORF at the 5' end and 9 structural ORFs at the 3' end (Brinton et al., unpublished data; Zeng et al., 1995; Godeny et al., 1995; Smith, 1997). Both coronaviruses and arteriviruses produce a 3' co-terminal nested set of mRNAs during their replication cycle (Plagemann, 1996; Lai, 1990). Each SHFV subgenomic mRNA contains a leader sequence at its 5 ' end that is identical to the one located near the 5' end of the genome (Zeng et al., 1995). Short conserved sequences, designated junction sequences, precede each ORF (Zeng et al., 1995; Smith, 1997). SHFV junction sequences are shorter and less redundant than coronavirus intergenic sequences.

It was previously reported that a $35 / 38 \mathrm{kDa}$ protein in $\mathrm{DBT}$ cytoplasmic extracts binds to the 3' end of the negative-strand RNA of the coronavirus, MHV (Furuya and Lai, 1993) and that three cellular proteins, 70,48 , and $35 / 38 \mathrm{kDa}$, bind to the MHV negativestrand intergenic region RNA located between genes 6 and 7 (Zhang and Lai, 1995). In this study the 3' NCR of the SHFV negative-strand RNA was used as a probe in gel mobility shift, UV-induced cross-linking, and northwestern blotting assays with cytoplasmic extracts from uninfected and SHFV-infected MA104 cells to determine whether any cellular or viral proteins bind to this RNA.

\section{MATERIALS AND METHODS}

\subsection{Cells and Virus}

Cytoplasmic extracts were prepared as described previously (Blackwell and Brinton, 1995) from confluent monolayers of MA104 cells that had been cultured in Dulbecco's minimal essential medium supplemented with $10 \%$ fetal bovine serum. For preparation of infected cell extracts, MA104 cells were infected with SHFV, strain LVR 42-0/M6941, at a multiplicity of infection of 5 for $6 \mathrm{hr}$ prior to cell lysis . Virus stock pools were prepared in MA104 cells and contained titers of about $10^{8} \mathrm{PFU} / \mathrm{ml}$.

\subsection{Construction of cDNA Templates and in Vitro Synthesis of RNA Transcripts}

SHFV RNA was purified from virions using Catrimox-14 Surfactant (Iowa Biotechnology Corp.). SHFV 3'(-)NCR RNA cDNA was synthesized from viral RNA by reverse transcription (RT), amplified by PCR and cloned into the pCR 2.1 plasmid (Invitrogen). Different primer pairs were used to generate the PCR templates for the various truncated SHFV 3'(-) RNAs used as probes. All antigenomic sense primers contained the T7 pro- 
moter. Negative-strand RNA transcripts were synthesized in vitro from the various PCR templates with T7 RNA polymerase. In vitro RNA transcription reactions were performed as described by the manufacturer (Ambion).

\subsection{Gel Mobility Shift Assay}

${ }^{32}$ P-labeled SHFV 3'(-)NCR RNA and uninfected MA104 S100 cytoplasmic extracts were incubated for $30 \mathrm{~min}$ at room temperature in a volume of $10 \mu \mathrm{l}$ and then analyzed on nondenaturing polyacrylamide gels as described previously (Blackwell and Brinton, 1995).

\subsection{UV-Induced Cross-Linking Assay}

Binding reactions were done as described above and then the reaction mixtures were irradiated for $30 \mathrm{~min}$ with UV light on ice as described previously (Blackwell and Brinton, 1995). The cross-linked products were digested with RNase A, precipitated with $50 \%$ acetone/methanol, pelleted by microcentrifugation, and visualized by autoradiography.

\section{RESULTS}

\subsection{Analysis of Interactions between SHFV 3'(-)NCR RNA and MA104 Cytoplasmic Proteins}

To determine whether any MA104 cytoplasmic proteins bind specifically to the 3' end of the SHFV negative-strand RNA, gel mobility shift assays were performed. The RNA probe used in the initial experiments was 209 nucleotides (nt) in length and contained the entire 3' noncoding region of SHFV 3'(-)NCR (Zeng et al., 1995). Two RNAprotein complexes were detected in both mock- and SHFV-infected MA104 cytoplasmic extracts in gel mobility shift assays. The number and eletrophoretic mobility of the complexes formed between SHFV 3'(-)NCR RNA and MA104 proteins in mock- and SHFVinfected cytoplasmic extracts were identical, suggesting that these RNA-protein complexes contained only cellular proteins. To determine the specificity of these RNAprotein interactions, competition gel mobility shift assays were performed. Unlabeled SHFV 3'(-)NCR RNA was used as the specific competitor and poly (I)- poly (C), yeast tRNA, and an RNA synthesized in vitro from plasmid pCR 2.1 vector DNA were used as non-specific competitors. While $350 \mathrm{ng}$ of the specific competitor completely inhibited RNA-protein complex formation, no inhibition was detected with much higher amounts of poly (I)-poly (C), yeast tRNA, or plasmid RNA.

\subsection{Determination of the Molecular Masses of the Cellular Proteins That Bind to the SHFV 3'(-) NCR RNA}

UV-induced cross-linking and Northwestern blotting assays were utilized to determine the molecular masses of the cellular proteins that bind to the SHFV 3'(-)NCR RNA. Four MA104 cytoplasmic proteins $(103,86,55$, and $36 \mathrm{kDa})$ were detected with the ${ }^{32} \mathrm{P}$ labeled SHFV 3'(-)NCR by UV-induced cross-linking assays (Figure 1). Four cellular proteins with identical molecular masses were detected with the SHFV 3'(-)NCR probe in uninfected and SHFV-infected cells extracts. 


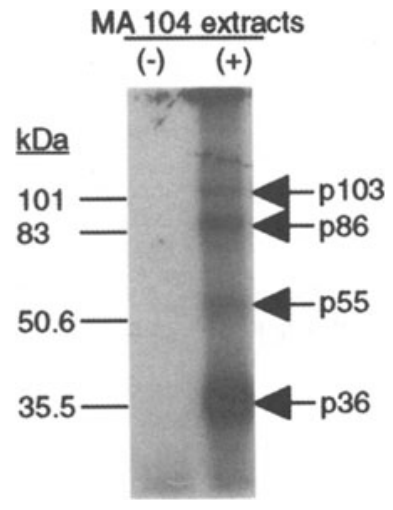

Figure 1. UV-induced cross-linking analysis of cellular proteins that bind to the SHFV 3'(-)NCR RNA. ${ }^{32}$ P-labeled SHFV 3'(-)NCR RNA probe was incubated with MA104 S100 cytoplasmic extract $(5 \mu \mathrm{g})$ for $30 \mathrm{~min}$ at room temperature and the RNA-protein complexes were subjected to UV-induced cross-linking. The arrows indicate the cellular protein bands $(103,86,55$, and $36 \mathrm{kDa}) .(-)$, no cellular extracts added; (+), MA104 S100 cytoplasmic extracts added to the reaction. Bio-Rad protein standards were used as markers.

Only three of the cellular proteins, 103,55 , and $36 \mathrm{kDa}$, were detected in northwestern blotting assays. The $86 \mathrm{kDa}$ protein may not renature after separation by SDS-PAGE, may not transfer efficiently, may require a co-factor to bind to the SHFV 3'(-)NCR RNA or may require another protein for cooperative binding.

\subsection{Mapping the Binding Sites of the Four Cellular Proteins on the SHFV 3'(-)NCR RNA}

SHFV 3'(-)5-184 RNA and SHFV 3'(-)45-184 RNA (Figure 2) were generated and used as competitors in competition gel mobility shift assays with the ${ }^{32} \mathrm{P}$-labeled SHFV 3'(-)209 RNA probe. The data obtained indicated that the 5' portion of the SHFV 3'(-) NCR RNA contained binding sites for each of the four cellular proteins. Additional truncated SHFV 3'(-)NCR RNAs were then generated and used as probes in UV-induced cross-linking assays to more precisely locate the binding sites for the individual cellular proteins. ${ }^{32}$ P-labeled SHFV 3'(-)5-184 RNA and SHFV 3'(-)137-202 RNA probes were used in initial UV-induced cross-linking assays. While the binding sites for the four proteins $(103,86,55$, and $36 \mathrm{kDa})$ were detected with the SHFV 3' (-)5-184 RNA probe, only the binding sites for the 103 and $86 \mathrm{kDa}$ proteins and a partial binding site of the 55

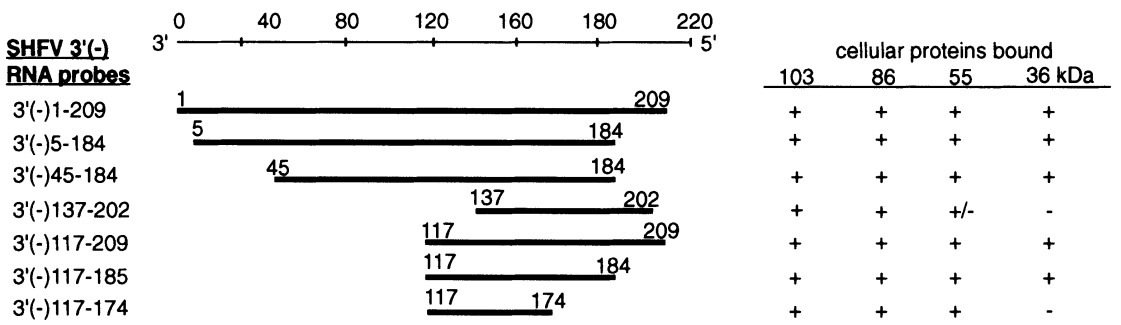

Figure 2. Schematic diagram of the truncated SHFV 3'(-)NCR RNAs used for mapping the binding sites of the cellular proteins on SHFV 3'(-) RNA. cDNAs representing the various truncated SHFV 3'(-) RNAs were generated by PCR and used as templates for in vitro RNA transcription. The nucleotides were numbered starting from the 3 end of the minus-strand RNA. The proteins detected by each of the deleted RNAs were assayed by UV-induced cross-linking. A summary of the data obtained is presented on the right. 
kDa protein were detected with the SHFV 3'(-)137-202 RNA probe. These data suggested that the region between nt 184 and 202 contains contact sites for the 36 and $55 \mathrm{kDa}$ proteins. An RNA which contained 20 additional 3' nt [SHFV 3'(-)117-209] bound efficiently to all four of the cellular proteins. To define the $5^{\prime}$ boundary of the binding region for the cellular proteins on the SHFV 3'(-)NCR RNA, SHFV 3'(-)117-184 RNA and SHFV 3'(-) 117-174 RNAs were generated and tested. While the SHFV 3'(-)117-184 RNA contained the binding sites for the all four of the proteins, SHFV 3'(-)117-174 RNA contained binding sites for only the 103,86 , and $55 \mathrm{kDa}$ proteins. Therefore, the binding sites for the $103,86,55$, and $36 \mathrm{kDa}$ proteins were mapped to the region between $\mathrm{nt} 117$ and 184 on the SHFV 3'(-)NCR RNA. Data obtained from the UV-induced cross-linking assays are summarized in Figure 2.

\subsection{Conservation of the MA104 Cellular Protein Binding Sites in Another Arterivirus 3'(-)NCR RNA}

The family Arteriviridae is currently composed of four members, equine arteritis virus (EAV), porcine reproductive and respiratory syndrome virus (PRRSV), lactate dehydrogenase-elevating virus (LDV) and SHFV. Natural infections with each of the arteriviruses are host restricted. To determine whether a divergent arterivirus can bind to the same MA104 cellular proteins as the SHFV 3'(-)NCR RNA, the 3' NCR of LDV-C negative-strand RNA [LDV-C 3'(-)NCR RNA] was used as an unlabeled competitor in competition gel mobility shift assays and as a labeled probe in UV-induced cross-linking assays with MA104 S100 cytoplasmic extracts (data not shown). The formation of SHFV RNA-protein complexes was inhibited by the unlabeled LDV-C 3'(-)NCR RNA competitor. In UV-induced cross-linking assays, the LDV-C 3'(-)NCR RNA probe detected four cellular proteins with identical molecular masses to those detected by the SHFV $3^{\prime}(-)$ NCR RNA.

\section{DISCUSSION}

We have demonstrated that cellular proteins in MA104 cytoplasmic extracts bind specifically to the SHFV 3'(-)NCR RNA. The molecular masses of the cellular proteins that bind to the SHFV 3'(-)NCR RNA in mock-infected and SHFV-infected MA104 cytoplasmic extracts are $103,86,55$, and $36 \mathrm{kDa}$, indicating that the proteins involved in the formation of these RNA-protein complexes are cellular proteins and not viral proteins. Using a series of 3' and 5' deleted, in vitro transcribed SHFV 3'(-)NCR RNAs, the binding sites for the four cellular proteins were mapped to the region between nucleotides 117 and 184. Competition gel mobility shift and UV-induced cross-linking analyses with LDV-C 3'(-)NCR RNA showed that this RNA contains binding sites for the same four cellular proteins as the SHFV 3'(-)NCR RNA. These results suggest that the 3'(-)NCR RNAs of divergent arteriviruses bind to the same conserved domains on cellular proteins in their respective host cells. The functions provided by the cellular proteins that bind to the SHFV 3'(-)NCR RNA are not yet known.

Recently, we have developed an in vivo SHFV replication system based on that of Hiscox et al. (1995) to determine whether the region containing the cellular protein binding sites (nt 117 to 184) on the SHFV 3'(-)NCR RNA functions as a cis-acting sequence for positive-strand RNA synthesis. Previous data obtained with other viral systems have been shown that the RNA region to which cellular proteins bind is required for viral multi- 
plication (Sriskanda et al., 1996; Yu and Leibowitz, 1995). Preliminary data obtained from our system indicates that the SHFV 3'(-)NCR RNA is required in cis for in vivo replication of an in vitro transcribed SHFV-CAT negative-strand RNA. Studies are underway to map the cis-acting sequence elements on the SHFV 3'(-)NCR.

\section{ACKNOWLEDGMENTS}

This research was supported by the Georgia State University Research Foundation.

\section{REFERENCES}

Blackwell J. L., and Brinton M. A., 1995, BHK cell proteins that bind to the 3' stem-loop structure of the West Nile virus genome RNA, J. Virol. 69: 5650-5658.

Furuya T., and Lai M. M. C., 1993, Three different cellular proteins bind to complementary sites on the 5'-endpositive and 3'-end-negative strands of mouse hepatitis virus RNA, J. Virol. 67: 7215-7222.

Godeny E. K., Zeng L., Smith S. L., and Brinton M. A., 1995, Molecular characterization of the 3' terminus of the simian hemorrhagic fever virus genome, J. Virol. 69: 2679-2683.

Gravell M., London W.T., Rodriguez M., Palmer A.E., and Hamilton R.S., 1980, Simian hemorrhagic fever (SHF): new virus isolate from a chronically infected patas monkey, J. Virol. 51: 99-106.

Hiscox J.A., Mawditt,K.L., Cavanagh D., and Britton P., 1995, Investigation of the control of coronavirus subgenomic mRNA transcription by using T7-generated negative-sense RNA, J.Virol 69: 6219-6227.

Lai, M.M.C., 1990, Coronavirus: organization, replication, and expression of genome. Annu. Rev. Microbiol. 44: 303-333.

London W.T., 1977, Epizootology, transmission and approach to prevention of fatal simian hemorrhagic fever virus in rhesus monkeys, Nature 268: 344-345.

Plagemann P.G.W., 1996, Lactate dehydrogenase-elevating virus and related viruses, in: Virology, Third Edition, (Fields, B.N., Knipe, D.M., Howley P.M., et al., eds.), Lippincott-Raven Publishers, Philadelphia, PA, pp 1075-1093.

Sagripanti J.L., 1984, The genome of simian hemorrhagic fever virus, Arch. Virol. 82: 61-72.

Sagripanti J.L, Zandomeni R.O., and Weinmann R., 1986, The cap structure of simian hemorrhagic fever viron RNA, Virology 151: 146-150.

Smith, S. L., Wang, X. C., and Godeny, E. K., 1997, Organization of the simian hemorrhagic fever virus (SHFV)genome and identification of the sgRNA junction sequences, Gene. In press.

Sriskanda V.S., Pruss G., Ge X., and Vance V.B., 1996, An eight-nucleotide sequence in the potato virus X 3'untranslated region is required for both host protein binding and viral multiplication, J. Virol. 70: 5266-5271.

Yu W., and Leibowitz J.L., 1995, A conserved motif at the 3' end of mouse hepatitis virus genomic RNA is required for host protein binding and viral RNA replication, Virology 214: 128-138.

Zeng L., Godeny E.K., Methven S.L, and Brinton M.A., 1995, Analysis of simian hemorrhagic fever virus (SHFV) subgenomic RNAs, junction sequences, and 5' leader, Virology 207: 543-548.

Zhang, X., and Lai M.M.C., 1995, Interaction between cytoplasmic proteins and the intergenic (promoter) sequence of mouse hepatitis virus RNA: correlation with the amounts of subgenomic mRNAs transcribed, $J$. Virol. 69: 1637-1644. 